\title{
Biodegradable Polymersomes for the Delivery of Gemcitabine to Panc-1 Cells
}

\author{
Nimil Sood, ${ }^{1}$ Walter T. Jenkins, ${ }^{2}$ Xiang-Yang Yang, ${ }^{2}$ Nikesh N. Shah, ${ }^{3}$ \\ Joshua S. Katz, ${ }^{4}$ Cameron J. Koch, ${ }^{2}$ Paul R. Frail, ${ }^{5}$ Michael J. Therien, ${ }^{6}$ \\ Daniel A. Hammer, ${ }^{1,4}$ and Sydney M. Evans ${ }^{2}$ \\ ${ }^{1}$ Department of Chemical and Biomolecular Engineering, University of Pennsylvania, Philadelphia, PA, USA \\ ${ }^{2}$ Department of Radiation Oncology, University of Pennsylvania, Perelman School of Medicine, Philadelphia, PA, USA \\ ${ }^{3}$ Department of Neuroscience, University of Miami, Coral Gables, FL, USA \\ ${ }^{4}$ Department of Bioengineering, University of Pennsylvania, Philadelphia, PA, USA \\ ${ }^{5}$ Department of Chemistry, University of Pennsylvania, Philadelphia, PA, USA \\ ${ }^{6}$ Department of Chemistry, Duke University, Durham, NC, USA
}

Correspondence should be addressed to Sydney M. Evans; sydevans@mail.med.upenn.edu

Received 23 February 2013; Accepted 22 April 2013

Academic Editor: Umesh Gupta

Copyright (C) 2013 Nimil Sood et al. This is an open access article distributed under the Creative Commons Attribution License, which permits unrestricted use, distribution, and reproduction in any medium, provided the original work is properly cited.

\begin{abstract}
Traditional anticancer chemotherapy often displays toxic side effects, poor bioavailability, and a low therapeutic index. Targeting and controlled release of a chemotherapeutic agent can increase drug bioavailability, mitigate undesirable side effects, and increase the therapeutic index. Here we report a polymersome-based system to deliver gemcitabine to Panc-1 cells in vitro. The polymersomes were self-assembled from a biocompatible and completely biodegradable polymer, poly(ethylene oxide)-poly(caprolactone), PEOPCL. We showed that we can encapsulate gemcitabine within stable $200 \mathrm{~nm}$ vesicles with a $10 \%$ loading efficiency. These vesicles displayed a controlled release of gemcitabine with $60 \%$ release after 2 days at physiological pH. Upon treatment of Panc- 1 cells in vitro, vesicles were internalized as verified with fluorescently labeled polymersomes. Clonogenic assays to determine cell survival were performed by treating Panc-1 cells with varying concentrations of unencapsulated gemcitabine (FreeGem) and polymersomeencapsulated gemcitabine (PolyGem) for 48 hours. $1 \mu \mathrm{M}$ PolyGem was equivalent in tumor cell toxicity to $1 \mu \mathrm{M}$ FreeGem, with a one log cell kill observed. These studies suggest that further investigation on polymersome-based drug formulations is warranted for chemotherapy of pancreatic cancer.
\end{abstract}

\section{Introduction}

Pancreatic adenocarcinoma is the fourth highest cause of cancer death with a 5-year survival rate of less than $6 \%$ [1]. Despite the use of surgery, radiation, and/or chemotherapy [2], local recurrence and metastasis invariably occur. The causes of resistance of pancreatic tumors are not completely understood. The inability to deliver adequate adjuvant therapy due to local normal tissue toxicity, limitations caused by tumor microenvironment (hypoxia, $\mathrm{pH}$ ), and active drug export out of tumor cells likely cause this resistance [3, 4]. Modifications to the delivery of chemotherapeutics that improve the therapeutic ratio (TR) are highly desirable in order to allow higher drug delivery while minimizing toxicity to normal tissues.

Gemcitabine is a commonly used water soluble anticancer agent that acts as an antimetabolite; it is considered an efficacious addition to radiation therapy in pancreatic cancer [5]. Gemcitabine is an S-phase deoxycytidine analog $\left(2^{\prime}, 2^{\prime}\right.$ difluorodeoxycytidine). Its mechanism of action involves competitive incorporation into DNA, masked termination (causing termination of DNA synthesis without being excised out of the strand), and self-potentiation (promoting its own activity by inhibiting regulatory enzymes involved in DNA synthesis). Like most chemotherapeutics, its use has significant limitations. Gemcitabine is rapidly metabolized 
in the blood stream with a short plasma half-life (for short infusions, 32-94 min) and has substantial side effects that limit the dose that can be given, especially when combined with radiation therapy [6]. In a phase 1 study, concurrent application of gemcitabine and radiation caused nausea, vomiting, dehydration, and gastric ulceration resulting in a $44 \%$ hospital admission rate [7]. These side effects are much greater for concurrent therapy than for just radiation, which has been linked primarily to nausea. Encapsulation of gemcitabine in a carrier vehicle has the potential to reduce dose-limiting side effects while improving the drug delivery to the tumor. The latter includes increased circulation time and preferential accumulation in tumor due to the enhanced permeability and retention (EPR) effect [8].

Encapsulation of gemcitabine to address the challenges of rapid blood metabolism and low therapeutic ratio has been previously investigated. In one study, gemcitabine loaded in sonochemically prepared bovine serum albumen (BSA) microspheres was evaluated for cell killing in renal cancer in vitro [9]. These microspheres exhibited poor dynamics of release and were unable to take advantage of the EPR effect observed in solid tumors due to their large size $(\sim 1 \mu \mathrm{m}$ diameter), an effect which requires $150-300 \mathrm{~nm}$ particles in diameter [8]. In another study, albumin nanospheres were loaded with gemcitabine [10]; this delivery system also had poor release kinetics with $100 \%$ of the drug being released in 24 hours. In a third study, encapsulated gemcitabine within poly(ethylene glycol)-poly(DL-lactic acid) (PEG-PDLLA) nano vesicles showed toxicity against SW1990 pancreatic cells [11]. The vesicle morphology and size of these vesicles were very variable.

Polymer vesicles, or polymersomes, are self-assemblies of amphiphilic block copolymers that can encapsulate both hydrophobic and hydrophilic compounds [12, 13]. Their highly tunable chemistry allows for diverse functionalities and applications [14]. Polymersomes possess superior biomaterial properties compared to their lipid counterparts (liposomes) including greater stability, storage capacity, release characteristics, and plasma circulation times [15-18]. The hydrophilic block is often composed of poly(ethylene oxide) (PEO) head groups, which helps reduce non-specific interactions with blood proteins due to their hydrophilicity and steric hindrance effects. This greatly reduces opsonization of nanoparticles and increases their plasma circulation time. Several biodegradable hydrophobic blocks can be utilized for drug delivery including polycaprolactone (PCL) and polylactide (PLA) for polymersomes and polylacticco-glycolic acid (PLGA) for nanoparticles [14, 19-21]. PCL has several advantages over the other polymers including high permeability to small molecules, maintenance of neutral $\mathrm{pH}$ after degradation, ease of blending with other polymer blocks, and long-term and tunable erosion kinetics [22].

Recognizing the potential of PEO-PCL polymersomes for use in cancer treatment, this paper describes the novel use of PEO-PCL nanopolymersomes for gemcitabine encapsulation and in vitro delivery to Panc-1 cells. We investigated the polymersome release kinetics of gemcitabine, vesicle internalization by Panc-1 cells, and cell toxicity of PolyGems compared to standard gemcitabine (FreeGem). Polymersomes were internalized by Panc- 1 cells and had equivalent cell toxicity at the same total dose when loaded with gemcitabine. These results suggest that PolyGems have the potential to be an attractive route to improve gemcitabine delivery in vivo.

\section{Materials and Methods}

2.1. Materials. Gemcitabine (Gemzar) was obtained from Eli Lilly and Company (Indianapolis, IN). Panc-1 cells were obtained from the ATCC. DMEM/F12 Ham's (50/50) without phenol red was purchased from Zen-Bio (Research Park, NC). The meso-to-meso ethyne-bridged (porphinato) zinc (II) trimer $\left(\mathrm{PZn}_{3}\right)$ with a 9-methoxy-1,4,7-trioxanonyl substituent on one aryl group and a more hydrophobic 3,3dimethyl-1-butyloxy substituent on the other was synthesized as previously described [23]. Glacial acetic acid, methylene chloride, methylene blue for colony staining, phosphate buffered saline (PBS), sodium acetate trihydrate, and sodium chloride were purchased from Fisher Chemicals (Pittsburgh, PA). Polycarbonate extrusion membranes $(13 \mathrm{~mm})$ were purchased from Whatman (Piscataway, NJ). Centrifugal filter units were purchased from Millipore (Billerica, MA). Dialysis cassettes were purchased from Spectrum Laboratories (Rancho Dominguez, CA).

2.2. PEO-PCL Preparation. PEO-b-PCL with 45 and 105 monomer repeat units per block, respectively, $(\mathrm{MW}=14,00$ $\mathrm{g} \mathrm{mol}^{-1}$ ) was synthesized prior to this work [14]. The block copolymer was generated via ring-opening polymerization of cyclic $\varepsilon$-caprolactone. Briefly, monomethoxypoly(ethylene oxide) (2k) was filled in a flame-dried flask under argon. Caprolactone monomer was injected into the flask via syringe, and two drops of stannous (II) octoate was added to the reaction mixture. The reaction occurred at $130^{\circ} \mathrm{C}$ for 24 hours. The copolymer was isolated by dissolving the product in methylene chloride and precipitating in excess methanol/hexane at $4^{\circ} \mathrm{C}$. The resulting powder was dried further. The block copolymer was purified via gel permeation chromatography, and the molecular weight was determined by ${ }^{1} \mathrm{H}$ NMR.

2.3. Vesicle Preparation. Polymersomes were synthesized by the thin film hydration method as described elsewhere [14]. Briefly, $200 \mu \mathrm{L}$ of a $100 \mathrm{mg} / \mathrm{mL}$ PEO-PCL solution in methylene chloride were deposited on a roughened teflon strip and allowed to dry overnight under vacuum. A $2.21 \mathrm{mg} / \mathrm{mL}$ (corresponding to $5: 1$ molar ratio of drug : polymer) solution lyof gemcitabine in $0.9 \%$ saline ( 290 mOsm) was added to the film in a $20 \mathrm{~mL}$ vial in order to hydrate the polymer. Polymersomes were formed by one hour of sonication at $60^{\circ} \mathrm{C}$ and 5 freeze/thaw cycles using liquid nitrogen. A narrow size distribution of polymersomes was obtained by successive extrusion through $400 \mathrm{~nm}, 200 \mathrm{~nm}$, and $100 \mathrm{~nm}$ membranes using a thermobarrel extruder (Lipex Biomembranes, Vancouver, Canada) operating at $65^{\circ} \mathrm{C}$. Size was verified using 
a Nano Zs Zetasizer (Malvern Instruments, Southborough, MA). $0.9 \%$ saline without drug was used as the hydration solution for control studies. For cellular uptake studies, polymersomes were loaded with the porphyrin-based nearIR fluorophore, $\mathrm{PZn}_{3}\left(\lambda_{\mathrm{ex}}=785 \mathrm{~nm}, \lambda_{\mathrm{em}}=800 \mathrm{~nm}\right)$, at a molar ratio of $40: 1$ polymer to $\mathrm{PZn}_{3}$ by cocasting the $\mathrm{PZn}_{3}$ with the polymer film. Before cell culture studies, vesicles were sterilized for 30 minutes via germicidal UV irradiation.

2.4. Cryo-TEM. Cryogenic transmission electron microscopy was performed at the University of Pennsylvania in the Penn Regional Nanotechnology Facility (Philadelphia, PA). Lacey formvar/carbon grids (Ted Pella) were rinsed with chloroform to remove the formvar template and subsequently carbon coated with a Quorum Q150T ES carbon coater (Quorum Technologies, UK). Grids were cleaned with hydrogen/oxygen plasma for 15 seconds using the Solarus Advanced Plasma System 950 (Gatan, Pleasanton, CA). Polymersome sample $(2 \mu \mathrm{L})$ was deposited on lacey formvar/carbon mesh grid (Ted Pella) and inserted into a cryoplunger (Gatan Cp3, Gatan). The sample was blotted by hand and plunged into liquid ethane. Samples were transferred to a cryoholder (Gatan CT3500TR, Gatan), and the cryoholder was immediately inserted into a JEOL 2010 TEM (JEOL) operating at $200 \mathrm{kV}$. Micrographs were imaged with an Orius SC200 digital camera.

2.5. Vesicle Release Kinetics. Nanovesicles were prepared as described above. After extrusion, vesicles were concentrated to $0.5 \mathrm{~mL}$ volume using $3 \mathrm{kDa}$ centrifugal filters made from regenerated cellulose. Additional removal of unencapsulated drug was performed via dialysis against a $\mathrm{pH} 5.0$ sodium acetate/sodium chloride buffer (acidified with glacial acetic acid) or pH 7.4 PBS buffer. Immediately following dialysis, $250 \mu \mathrm{L}$ sample aliquots were placed in microdialysis tubes and stored in $22 \mathrm{~mL}$ of $\mathrm{pH} 5.0$ or 7.4 buffer in a $37^{\circ} \mathrm{C}$ oven. At predefined time points, aliquots were taken from the buffer solution and read on a UV/Vis spectrophotometer at $270 \mathrm{~nm}\left(\varepsilon=9.86 \times 10^{-3} \mathrm{~cm}^{-1} \mu \mathrm{M}^{-1}\right)$. Aliquots were returned to the buffer to maintain a constant volume. $100 \%$ release was determined by addition of $100 \mu \mathrm{L}$ of a $1 \%$ Triton-X solution to the PolyGem vials after one week, and the gemcitabine absorbance after overnight storage at $37^{\circ} \mathrm{C}$ was measured.

2.6. Cell Culture. At the time of receipt from the ATCC, cells were placed in cryovials and stored in liquid nitrogen for future use. Cells for study were defrosted using standard procedures. New cell stock was defrosted at 6-month intervals. Panc-1 cells were cultured in DMEM/F12 Ham's (50/50) without phenol red and with $12 \%$ fetal calf serum and $1 \%$ Pen/Strep. Cells were maintained in T75 plastic culture flasks at $37^{\circ} \mathrm{C}$ in a humidified atmosphere containing $5 \%$ $\mathrm{CO}_{2}$ in air. Flasks were subcultured when they were 75$90 \%$ confluent. Five minute exposure to $0.05 \%$ trypsin-EDTA was used to release attached cells from the tissue culture surface.
2.7. In Vitro Cellular Uptake of Vesicles. Fluorescent polymersomes were synthesized as previously described. The concentration of $\mathrm{PZn}_{3}$ was determined by Beer's law using the established extinction coefficient $\varepsilon(795 \mathrm{~nm})=1.25 \mathrm{~cm}^{-1} \mu \mathrm{M}^{-1}$ [24]. Panc- 1 cells were plated in triplicate at 5,000 cells per well in 96 well (black frame, clear well) cell culture plates (Isoplate96 TC, Perkin Elmer). Cells were allowed to adhere overnight. The following day, varying concentrations of fluorescent polymersomes were added to wells and were incubated for 12, 24, and 48 hours. At each time point, one plate was removed from incubation washed three times with media to remove all polymersomes that were not internalized. Vesicles were illuminated on a LICOR Odyssey $\left(\lambda_{\mathrm{ex}}=488 \mathrm{~nm}\right.$, $\left.\lambda_{\mathrm{em}}=810 \mathrm{~nm}\right)$. A calibration curve was generated to relate the fluorescence signal from wells to the concentration of $\mathrm{PZn}_{3}$ to determine cellular uptake. Confocal laser scanning microscopy (CLSM) was used to visualize vesicle internalization in Panc-1 cells incubated with fluorescent polymersomes for 12 hours. An Olympus FluoView FV1000 confocal microscope (Center Valley, PA) with a PLFLN 40x oil objective lens was used to obtain $z$-stacks of cells with a scan speed of $8.0 \mu \mathrm{s} \mathrm{pixel}^{-1}$ and step size of $1 \mu \mathrm{m}$.

2.8. In Vitro Toxicity. Panc-1 cells were plated onto $60 \mathrm{~mm}$ tissue culture dishes. After 48 hours, the plates were examined under the microscope for evidence of cell growth. Only plates containing of $50-90 \%$ confluent cells were used for study. Following the removal of spent media, the plates were rinsed with $2 \mathrm{~mL}$ of fresh media. The rinsed media was then replaced with $2 \mathrm{~mL}$ of treatment solution (PolyGem, FreeGem, or blank polymersomes). Dishes were incubated for an additional 48 hours. At the time of assay, cells were removed from the plate by incubating $2 \mathrm{~mL}$ of $0.05 \%$ trypsin-EDTA with the cells for 5 minutes at $37^{\circ} \mathrm{C}$. Trypsin was inactivated using fresh media with serum, and the total cell number was determined using a Coulter counter. A clonogenic assay was performed using standard techniques [25]. Plates were incubated for two weeks, with the goal of obtaining 25-250 colonies per plate. The surviving fraction was determined by normalizing the colony count of the treated condition by the initial cell number plated. The concentration of gemcitabine as reported for PolyGem represents the total encapsulated concentration. We corrected for the drug release relative to time, which was $60 \%$ release at 48 hours. The survival graphs were presented in absolute fractions with the maximal plating efficiency of untreated Panc-1 cells being 0.60. All comparisons were made to this value. Qualitative morphology of treated cells was observed using a Zeiss inverted microscope. Panc-1 cells were treated with media, $5 \mu \mathrm{M}$ FreeGem, $5 \mu \mathrm{M}$ PolyGem, or $2.5 \mu \mathrm{M}$ blank polymersomes.

2.9. Statistical Analysis. All experiments were performed in triplicate, except where noted. In vitro toxicity studies were performed in duplicate with varying amounts of initial cell plating to ensure the resulting colonies were within a countable range (50-200 colonies). Data were reported as mean \pm standard deviation and analyzed by single-factor ANOVA, setting the level of significance at $P<0.05$. 


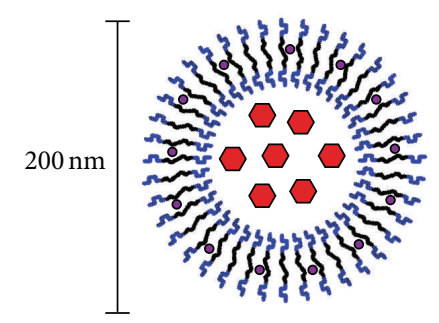<smiles>Nc1ccn(C2OC(CO)C(O)C3(F)C(=C4CCCCC4)OC23)c(=O)n1</smiles><smiles>CC#CC(C)OCCC(=O)OCC(C)(C)OCCCCCCOC(C)C</smiles>

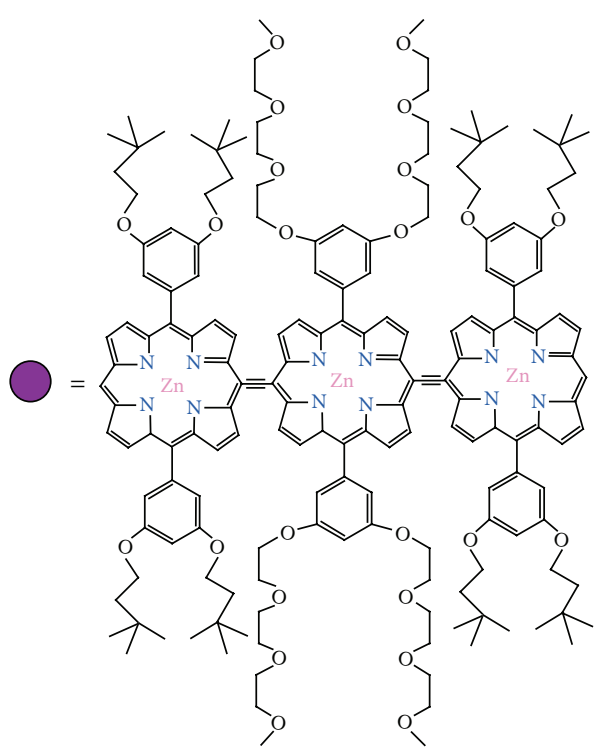

FIGURE 1: Schematic representation of PolyGem. In aqueous solution, poly(ethylene oxide)-b-poly(caprolactone) (PEO-PCL) selfassemble into spherical polymer vesicles (polymersomes), with the hydrophobic PCL chains orienting end to end to form the bilayer. The figure represents a uniaxial cross section of the polymersome, with gemcitabine $(\square)$ encapsulated in the aqueous lumen. Vesicles can also be made to include $\mathrm{PZn}_{3}(\mathrm{O})$ in the hydrophobic membrane.

\section{Results}

3.1. Vesicle Preparation and Characterization. Nanovesicles were prepared by thin-film hydration. This method is particularly attractive for making polymersomes for biological applications as it does not require the use of potentially toxic organic solvents during the hydration step (as is employed in the solvent injection method) [26].

Figure 1 shows a schematic of our novel carrier. The size distribution of vesicles was measured using dynamic light

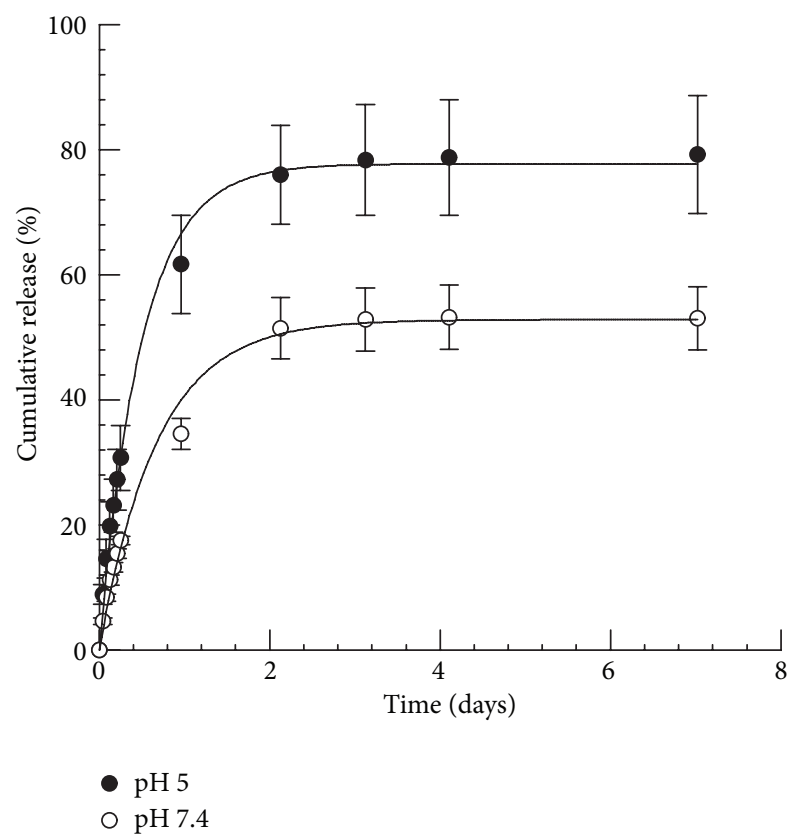

FIgure 2: Cumulative in situ release of gemcitabine from PolyGem at pH 5.0 and 7.4 and $37^{\circ} \mathrm{C}$, as measured via UV/Vis spectroscopy for 7 days. $n=3$ for each data point and error bars represent standard deviation.

TABLE 1: Hydrodynamic diameter and polydispersity of different vesicle formulations.

\begin{tabular}{lcc}
\hline Vesicle type & $D_{h}(\mathrm{~nm})$ & Polydispersity \\
\hline PolyGem & $180 \pm 12$ & $0.146 \pm 0.045$ \\
Blank polymersome & $181 \pm 13$ & $0.113 \pm 0.023$ \\
PZn $_{3}$-polymersome & $180 \pm 31$ & $0.163 \pm 0.020$ \\
\hline
\end{tabular}

scattering (DLS) and is shown in Table 1. Vesicles were serially extruded through $400,200,100,100$, and $200 \mathrm{~nm}$ polycarbonate membranes to reduce the sample polydispersity. The average hydrodynamic diameter of PolyGems was $180 \mathrm{~nm}$. The hydrodynamic diameter of blank polymersomes $(181 \mathrm{~nm})$ and $\mathrm{PZn}_{3}$-polymersomes $(180 \mathrm{~nm})$ did not statistically vary from PolyGems $(P>0.05)$. Vesicles were stored at $4^{\circ} \mathrm{C}$ and used in the studies within one week of preparation. We evaluated the stability of gemcitabine after sonication and freeze/thaw cycles by checking the absorbance of the drug before and after processing. There was no difference in the absorbance spectrum, indicating that the drug retained its structure.

3.2. Vesicle Drug Release. We explored the release profile of gemcitabine from polymersomes at $37^{\circ} \mathrm{C}$ at pH 5.0 and 7.4. The results are shown in Figure 2. Approximately $20 \%$ of the gemcitabine is released from the PolyGems within the first few hours at both pHs. The concentration of gemcitabine released begins to level off at approximately day two, indicating stable and controlled release after an initial period up to 48 hours. More gemcitabine is released per unit time at $\mathrm{pH}$ 5.0 than at $\mathrm{pH} 7.4$. 


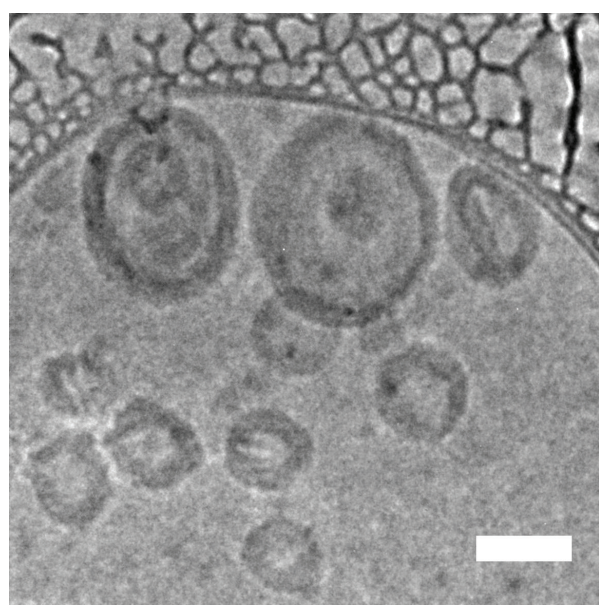

(a)

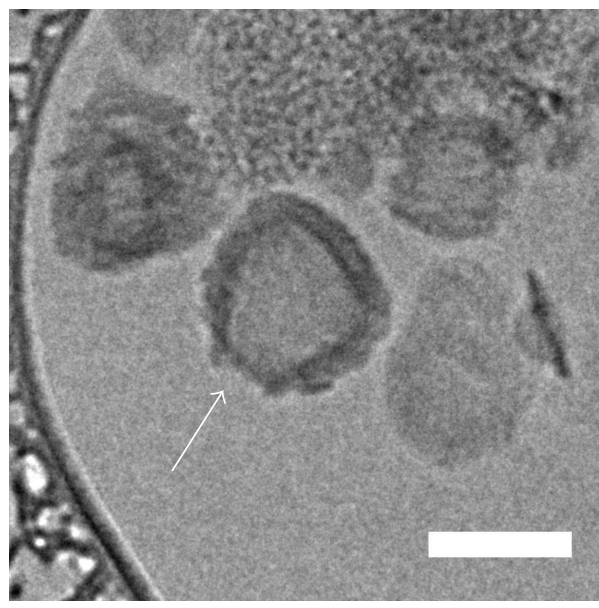

(c)

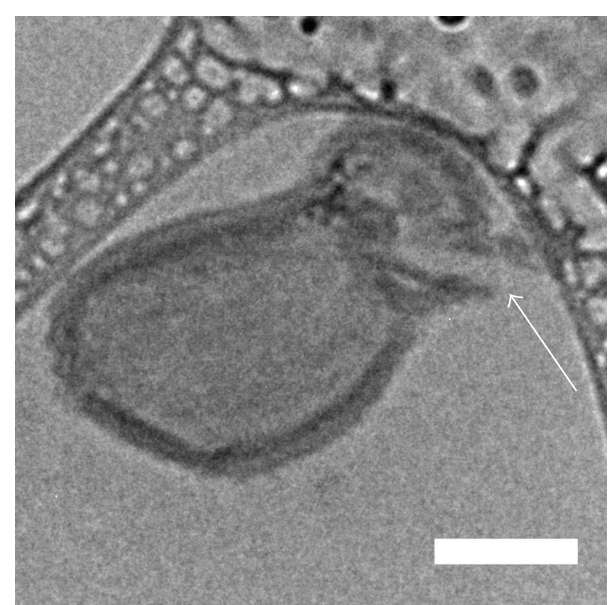

(b)

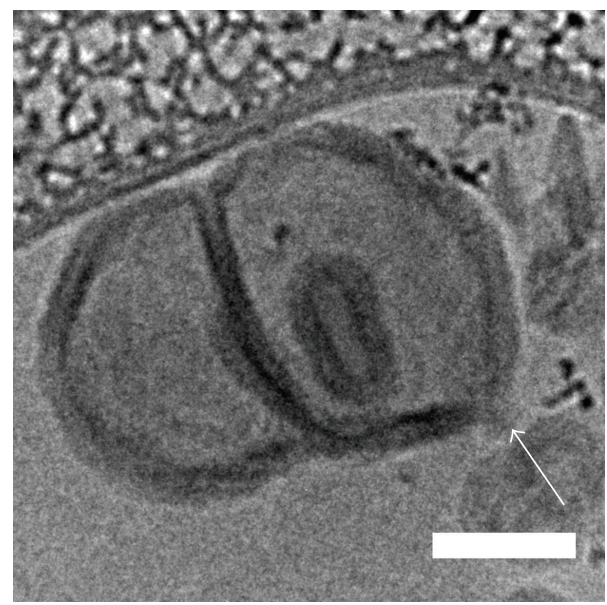

(d)

FIgURE 3: Cryo-TEM micrographs of PEO-PCL vesicles incubated for 12 hours. (a) pH 7.4. (b)-(d) pH 5.0. Arrows indicate areas of membrane degradation. Scale bar $=100 \mathrm{~nm}$.

The release curves are well fit by a function of the form $c / c_{0}=\alpha\left(1-e^{-t / \tau}\right)$, where $\alpha$ is a lumped constant and $\tau$ is the characteristic time constant. This functional form can be derived from a $1 \mathrm{D}$ analysis of drug diffusion across a semipermeable membrane. Fitting this equation to the release curves in Figure 2 reveals that $\tau_{\mathrm{pH} 5}$ is 12 hours and $\tau_{\mathrm{pH} 7}$ is 16 hours (lower values of $\tau$ indicate more rapid release).

3.3. Cryo-TEM of Vesicles. In order to understand the difference in observed release rate between vesicles at $\mathrm{pH} 5.0$ and 7.4, we observed the structure of nanovesicles using cryoTEM at the incubation different conditions. Figure 3 provides representative images of vesicles that have been incubated at either $\mathrm{pH} 7.4$ (Figure 3(a)) or $\mathrm{pH} 5.0$ (Figures 3(b)-3(d)) for 12 hours. The vesicles in Figure 3(a) have an even membrane thickness that is an indicative of an intact membrane. The vesicles in Figures 3(b)-3(d) have compromised membranes as shown by the arrows. In Figure 3(b), the membrane of one vesicle has completely disintegrated and has formed a pore.
Figure 3(c) shows a vesicle with a thinning portion of the membrane. Finally, in Figure 3(d) we see a membrane pore starting to form.

3.4. In Vitro Cellular Uptake. We visualized polymersome internalization by a cellular uptake study of blank polymersomes loaded with a hydrophobic porphyrin-based NIR fluorophore, $\mathrm{PZn}_{3}$, in the vesicle membrane. In our study, Panc-1 cells were incubated with 50, 250, or $500 \mathrm{nM}$ of $\mathrm{PZn}_{3}$ loaded polymersomes in 96-well plates for 12,24 , or 48 hours. A calibration was performed to relate the integrated intensity in wells to $\mathrm{PZn}_{3}$ concentration. Figure 4 shows the concentration of internalized $\mathrm{PZn}_{3}$ as a function of incubation time. Vesicle uptake increased with an increase in the concentration of $\mathrm{PZn}_{3}$ as well as incubation time. In order to confirm that the internalization from the uptake study was not surface association, Panc-1 cells were incubated with $500 \mathrm{nM} \mathrm{PZn} \mathrm{n}_{3}$ for 12 hours and imaged on a confocal microscope with $1 \mu \mathrm{m} z$-slices. Figure 4(c) shows $z$-slices 


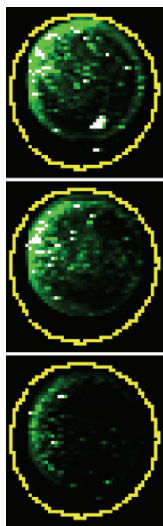

(a)

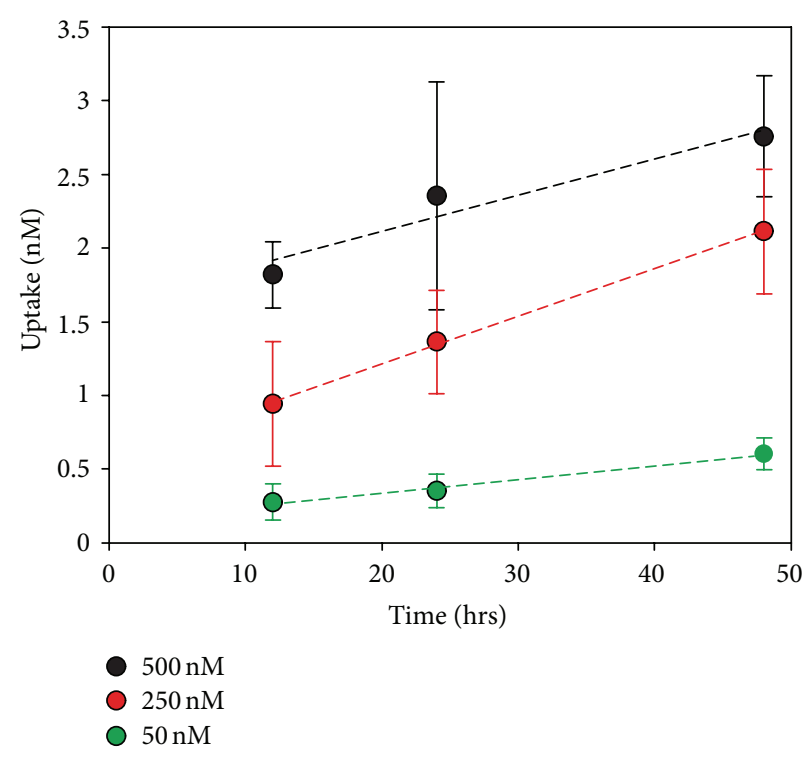

(b)

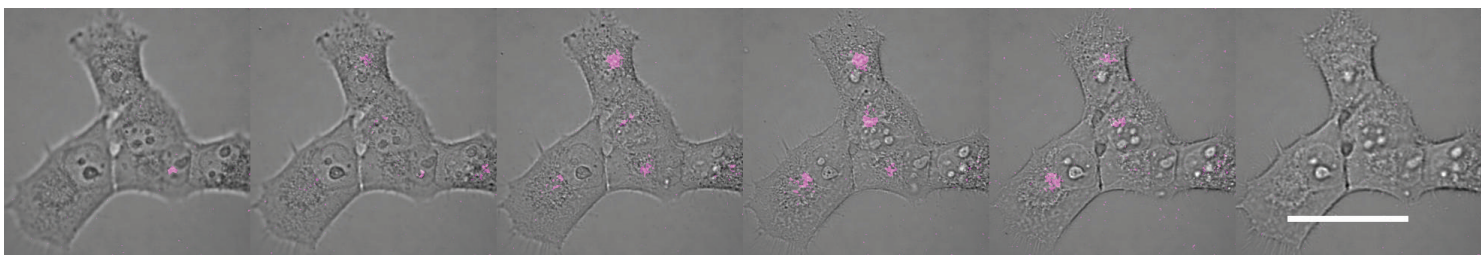

(c)

Figure 4: PolyGem internalization by Panc-1 cells. (a) Fluorescence intensity of $\mathrm{PZn}_{3}$-polymersomes internalized by cells in well plates corresponding to 48 hour time point. (b) Concentration of $\mathrm{PZn}_{3}$ uptake as a function of solution $\mathrm{PZn}_{3}$ concentration $(n=3)$. Error bars indicate standard deviation. (c) CLSM $z$-stack images of Panc-1 cells incubated with $\mathrm{PZn}_{3}$-polymersomes for 12 hours. Z-slices $(\Delta z=3 \mu \mathrm{m})$ are presented from left to right. Scale bar $=50 \mu \mathrm{m}$.

starting from the top of the cell and moving to the bottom. Vesicles are only observed internally and not on the cell surface.

3.5. In Vitro Cell Toxicity. Panc-1 cell survival was determined by a clonogenic assay following 48-hour FreeGem or PolyGem exposure using varying drug concentrations (Figure 5). Panc-1 survival is concentration dependent with an observed increase in cell kill as gemcitabine concentration was increased irrespective of the formulation used. A one $\log$ cell kill is observed at approximately $1 \mu \mathrm{M}$ gemcitabine, irrespective of the formulation. The only drug concentration where there was a significant difference in survival between FreeGem and PolyGems was at $0.05 \mu \mathrm{M}$ gemcitabine $(P=$ 0.048 ) where the PolyGem were more effective than the FreeGem. There was no significant cell kill using either blank polymersomes $(0 \mu \mathrm{M}$ PolyGem $)$ or media-only treatment $(0 \mu \mathrm{M}$ FreeGem $)$ as seen in Figure 5.

To qualitatively assess cell toxicity, Panc- 1 cells were imaged using differential interference contrast microscopy (DIC) following 48 hour exposure to media, $5 \mu \mathrm{M}$ FreeGem, $5 \mu \mathrm{M}$ PolyGem, or $2.5 \mu \mathrm{M}$ blank polymersomes. These doses of PolyGem, FreeGem, and blank polymersomes were chosen

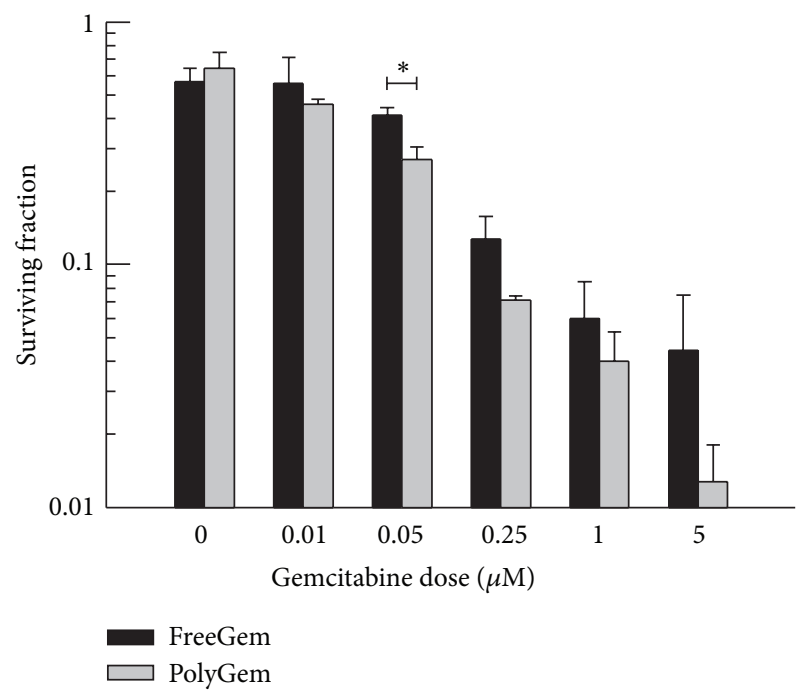

FIGURE 5: Panc-1 viability after 48 hour treatment with varying concentrations of FreeGem or PolyGem, as measured by a clonogenic assay. Error bars indicate standard deviation $(n=3) .{ }^{*} P<0.05$.

to reflect the maximum concentration of gemcitabine delivered to Panc-1 cells in the clonogenic assay and to equate 


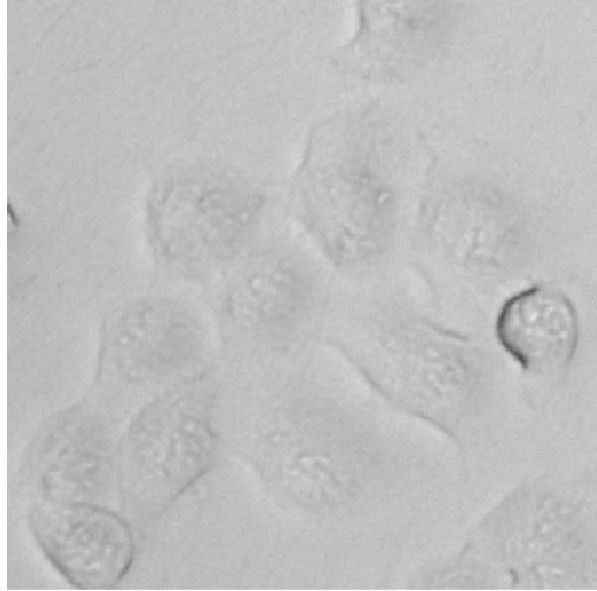

(a)

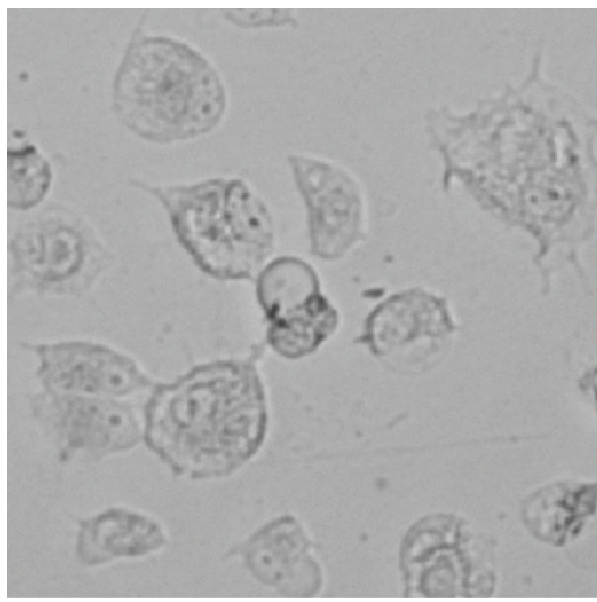

(c)

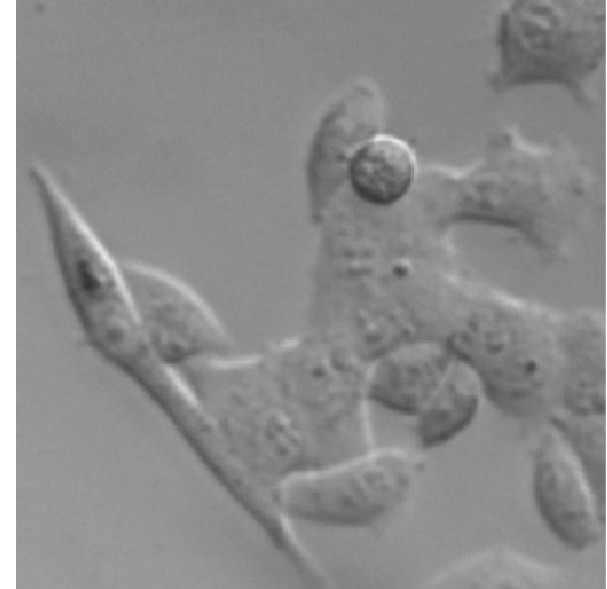

(b)

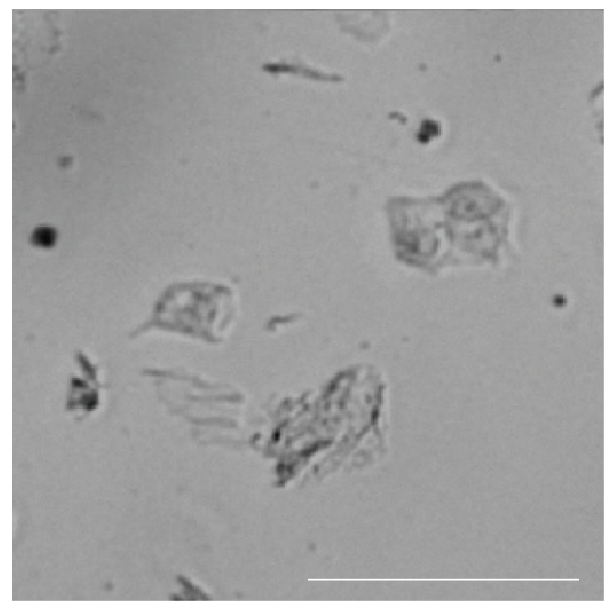

(d)

Figure 6: Cell phenotype as visualized by DIC. (a) Media control, (b) $2.5 \mu \mathrm{M}$ empty polymersomes, (c) $5 \mu \mathrm{M}$ FreeGem, and (d) $5 \mu \mathrm{M}$ PolyGem. Scale bar $=100 \mu \mathrm{m}$.

the concentration of PEO-PCL across the formulations $(2: 1$ ratio of drug to polymer). Figures 6(a) and 6(b) demonstrate that application either of media or blank polymersomes, respectively, has no effect on cell viability since the cells are morphologically normal. Both $5 \mu \mathrm{M}$ FreeGem and $5 \mu \mathrm{M}$ PolyGem treatments (Figures 6(c) and 6(d), resp.) resulted in significant cell killing as observed by the presence of morphologically abnormal cells and cellular debris.

\section{Discussion}

Despite the advancement of chemoradiation therapy for the treatment of pancreatic cancer, the 5-year survival rate continues to be among the lowest of solid tumors. Any improvement in the delivery of chemotherapeutics to pancreatic cancer should mitigate side-effects associated with treatment and improve the survival outlook for patients. In the studies reported herein, we created a prototype vehicle which could ultimately be capable of delivering gemcitabine, a potent pancreatic cancer drug, to pancreatic cancer. The major aim of this work was to perform a comparative in vitro study of the cell-killing efficacy of polymersomeencapsulated gemcitabine (PolyGem) and unencapsulated gemcitabine (FreeGem) on Panc-1 cells.

Bioresorbable polymersomes were synthesized from two FDA-approved polymers, poly(ethylene oxide) (PEO) and poly(caprolactone) (PCL). The polymers self-assembled in a fashion such that the hydrophobic PCL block was embedded in the polymersome membrane and the PEO block was exposed on the outer vesicle surface and inner corona (Figure 1). The internal hydrophilic lumen provided an aqueous environment for gemcitabine encapsulation, while hydrophobic porphyrin molecules were located within the membrane of the vesicles. Encapsulation of gemcitabine or porphyrin into the polymersomes did not alter the hydrodynamic diameter as compared to blank polymersomes (Table 1).

To improve the tumor-killing effect of drug delivery vesicles in vivo, maximal drug release should occur between 10 and 20 hours following injection, during which time the majority of vesicles have accumulated at the tumor and can cause toxicity [27]. In order to approximate the 
time constants of release of gemcitabine in vivo, we first explored the in situ release of gemcitabine from PolyGems under acidic and physiological conditions (Figure 2). A burst release of drug was observed during the first few hours, which was likely caused by the initially steep concentration gradient of gemcitabine present across the vesicle membrane, leading to a high initial diffusive flux. One explanation of this observation is that some entrapped gemcitabine in the outer PEO corona may have also contributed to the burst. Gemcitabine release was greater at all time points under acidic $\mathrm{pH}$ as compared to physiological pH. The PCL block is known to undergo hydrolysis of its ester linkages in solution, with a higher rate of degradation observed at acidic $\mathrm{pHs}$ as compared to physiological pHs [28, 29]. Consequently, both membrane permeability and the diffusive flux of gemcitabine increased at $\mathrm{pH}$ 5.0. The increased membrane permeability was verified with cryo-TEM images that show membrane degradation in the form of membrane pores and membrane thinning (Figure 3). Similar release curves under acidic and physiological conditions have been reported for doxorubicin release from PEO-PCL nanovesicles [14]. Understanding the release at $\mathrm{pH} 5.0$ is especially important for in vivo delivery to tumors because the extracellular environment of many tumors is acidic [30-32]. PolyGems would likely experience both acidic and physiological pHs as they reach the tumor.

The release curves were fitted to an exponential equation of the form $c / c_{0}=\alpha\left(1-e^{-t / \tau}\right)$ in order to extract the time constants $\tau_{\mathrm{pH} 5}$ and $\tau_{\mathrm{pH} 7}$. This functional form is consistent with passive diffusion across a membrane. These time constants describe the time required for $63 \%(1-1 / e)$ of the drug to be released due to diffusion and are functions of polymersome properties including geometric radius, membrane thickness, and diffusivity and partition coefficient of gemcitabine in the membrane. The time constants verify the quicker release observed at acidic conditions, with $\tau_{\mathrm{pH} 5}$ equal to 12 hours and $\tau_{\mathrm{pH} 7}$ equal to 16 hours. The ideal time constant for release depends on the time required for polymersomes to localize at a tumor site. Minimal drug should be released due to passive diffusion while the vesicles are still en route, and maximal and prolonged release should occur when vesicles have reached the tumor. As seen from the release curves, the majority of the gemcitabine was released within 2 days, which is consistent with the 10-20 hour window observed by Ahmed and coworkers [27].

While it is known that polymersomes will partition into tumor tissue in vivo [24], cellular internalization of the vesicles at the tumor is desirable so that the chemotherapeutic cargo can be released inside the cell in addition to the interstitial spaces where plasma clearance could be problematic. $\mathrm{PZn}_{3}$-based polymersomes have been utilized in the past to investigate polymersome uptake in dendritic cells (DCs) and DC trafficking in vivo $[33,34]$. The uptake study of PolyGems (Figure 4) showed that vesicle internalization is a concentration- and time-dependant process with more internalization occurring for higher polymersome number and longer incubation times. However, a relatively low percentage of vesicle internalization was observed. A likely contributor is the stealth character imparted by the
PEO block to the PolyGem, rendering the vesicles virtually invisible to Panc-1 cells. Another possible reason for low uptake could be a reduced cell surface area available for internalization due to cell adhesion on a tissue culture plate. Christian and coworkers observed a similar trend when incubating $\mathrm{PZn}_{3}$-polymersomes with dendritic cells. Negligible uptake was noticed for vesicles decorated with a PEO brush as compared to vesicles surface-conjugated with the HIV-derived TAT peptide [33]. Despite the low uptake without any targeting peptides, PolyGems performed at par with FreeGem (Figure 5). We believe that PolyGems released gemcitabine into the cell media, which was then internalized along with some vesicles via endocytosis and eventually leading to toxicity.

The true potential of PolyGems can best be determined in vivo where the drug kinetics are affected by all aspects of the tumor microenvironment and the therapeutic ratio (TR) can be assessed. This ratio is optimally greater than the one under in vivo conditions. Gemcitabine-based chemoradiotherapy in pancreatic cancer currently has a very narrow TR, being very close to one $[35,36]$. The result is substantial toxicity to the GI tract. We propose that this TR can be improved in future studies using the PolyGem technology. These nano-vesicles encapsulate and release gemcitabine over a prolonged period of time, which will likely be sufficient for nonspecific accumulation of PolyGems at the tumor site due to the EPR effect. A higher local concentration of drug in the tumor interstitial fluid can be expected because the polymersome prevents significant degradation of gemcitabine while trafficking to the tumor site. We predict that endocytosis of both free drug (due to release from the vesicles) and a portion of encapsulated drug will occur. The current findings-no decreased toxicity of gemcitabine using the encapsulated agent at equal concentrations of the free agent-are encouraging and support performing in vivo studies. Future studies will also focus on increasing efficacy by engineered internalization (i.e., using TAT-peptides conjugated to the polymersome surface) and targeting to specific cell surface receptors.

\section{Conflict of Interests}

The authors declare that they have no conflict of interests.

\section{Acknowledgments}

The authors acknowledge support from the NIH, R25-CA140116 (SME), CA115229 (DAH, MJT), and the NSF Materials Research Science and Engineering Centers (MRSEC) DMR05-20020 (DAH, MJT).

\section{References}

[1] A. Jemal, R. Siegel, J. Xu, and E. Ward, "Cancer statistics, 2010," CA Cancer Journal for Clinicians, vol. 60, no. 5, pp. 277-300, 2010.

[2] G. S. Group, "Radiation therapy combned with adriamycin or 5fluorouracil for the treatment of locally unresectable pancreatic carcinoma," Cancer, vol. 56, no. 11, pp. 2563-2568, 1985. 
[3] M. M. Gottesman, "Mechanisms of cancer drug resistance," Annual Review of Medicine, vol. 53, pp. 615-627, 2002.

[4] W. R. Wilson and M. P. Hay, "Targeting hypoxia in cancer therapy," Nature Reviews Cancer, vol. 11, no. 6, pp. 393-410, 2011.

[5] B. Pauwels, A. E. C. Korst, F. Lardon, and J. B. Vermorken, "Combined modality therapy of gemcitabine and radiation," Oncologist, vol. 10, no. 1, pp. 34-51, 2005.

[6] C. G. Moertel, S. Frytak, and R. G. Hahn, “Therapy of locally unresectable pancreatic carcinoma: a randomized comparison of high dose (6000 rads) radiation alone, moderate dose radiation (4000 rads + 5-fluorouracil), and high dose radiation +5 fluorouracil. The gastrointestinal tumor study group," Cancer, vol. 48, no. 8, pp. 1705-1710, 1981.

[7] R. A. Wolff, D. B. Evans, D. M. Gravel et al., "Phase I trial of gemcitabine combined with radiation for the treatment of locally advanced pancreatic adenocarcinoma," Clinical Cancer Research, vol. 7, no. 8, pp. 2246-2253, 2001.

[8] F. Alexis, E. Pridgen, L. K. Molnar, and O. C. Farokhzad, "Factors affecting the clearance and biodistribution of polymeric nanoparticles," Molecular Pharmaceutics, vol. 5, no. 4, pp. 505$515,2008$.

[9] O. Grinberg, A. Gedanken, C. R. Patra, S. Patra, P. Mukherjee, and D. Mukhopadhyay, "Sonochemically prepared BSA microspheres containing Gemcitabine, and their potential application in renal cancer therapeutics," Acta Biomaterialia, vol. 5, no. 8, pp. 3031-3037, 2009.

[10] J. M. Li, W. Chen, H. Wang et al., "Preparation of albumin nanospheres loaded with gemcitabine and their cytotoxicity against BXPC-3 cells in vitro," Acta Pharmacologica Sinica, vol. 30, no. 9, pp. 1337-1343, 2009.

[11] L. Jia, J. J. Zheng, S. M. Jiang, and K. H. Huang, "Preparation, physicochemical characterization and cytotoxicity in vitro of gemcitabine-loaded PEG-PDLLA nanovesicles," World Journal of Gastroenterology, vol. 16, no. 8, pp. 1008-1013, 2010.

[12] B. M. Discher, Y. Y. Won, D. S. Ege et al., "Polymersomes: tough vesicles made from diblock copolymers," Science, vol. 284, no. 5417, pp. 1143-1146, 1999.

[13] D. E. Discher and A. Eisenberg, "Polymer vesicles," Science, vol. 297, no. 5583, pp. 967-973, 2002.

[14] P. P. Ghoroghchian, G. Li, D. H. Levine et al., "Bioresorbable vesicles formed through spontaneous self-assembly of amphiphilic poly(ethylene oxide)-block-polycaprolactone," Macromolecules, vol. 39, no. 5, pp. 1673-1675, 2006.

[15] N. P. Kamat, G. P. Robbins, J. Rawson, M. J. Therien, I. J. Dmochowski, and D. A. Hammer, "A generalized system for photoresponsive membrane rupture in polymersomes," Advanced Functional Materials, vol. 20, no. 16, pp. 2588-2596, 2010.

[16] J. S. Katz, D. H. Levine, K. P. Davis, F. S. Bates, D. A. Hammer, and J. A. Burdick, "Membrane stabilization of biodegradable polymersomes," Langmuir, vol. 25, no. 8, pp. 4429-4434, 2009.

[17] J. S. Katz, S. Zhong, B. G. Ricart, D. J. Pochan, D. A. Hammer, and J. A. Burdick, "Modular synthesis of biodegradable diblock copolymers for designing functional polymersomes," Journal of the American Chemical Society, vol. 132, no. 11, pp. 3654-3655, 2010.

[18] G. P. Robbins, M. Jimbo, J. Swift, M. J. Therien, D. A. Hammer, and I. J. Dmochowski, "Photoinitiated destruction of composite Porphyrin-Protein polymersomes," Journal of the American Chemical Society, vol. 131, no. 11, pp. 3872-3874, 2009.
[19] W. Y. Ayen, K. Garkhal, and N. Kumar, "Doxorubicin-loaded (PEG)3-PLA nanopolymersomes: effect of solvents and process parameters on formulation development and in vitro study," Molecular Pharmaceutics, vol. 8, no. 2, pp. 466-478, 2011.

[20] F. H. Meng, G. H. M. Engbers, and J. Feijen, "Biodegradable polymersomes as a basis for artificial cells: encapsulation, release and targeting," Journal of Controlled Release, vol. 101, no. 1-3, pp. 187-198, 2005.

[21] J. Park, P. M. Fong, J. Lu et al., "PEGylated PLGA nanoparticles for the improved delivery of doxorubicin," Nanomedicine, vol. 5, no. 4, pp. 410-418, 2009.

[22] V. R. Sinha, K. Bansal, R. Kaushik, R. Kumria, and A. Trehan, "Poly- $\varepsilon$-caprolactone microspheres and nanospheres: an overview," International Journal of Pharmaceutics, vol. 278, no. 1, pp. 1-23, 2004.

[23] T. V. Duncan, K. Susumu, L. E. Sinks, and M. J. Therien, "Exceptional near-infrared fluorescence quantum yields and excitedstate absorptivity of highly conjugated porphyrin arrays," Journal of the American Chemical Society, vol. 128, no. 28, pp. 9000 9001, 2006.

[24] P. P. Ghoroghchian, P. R. Frail, K. Susumu et al., "Nearinfrared-emissive polymersomes: self-assembled soft matter for in vivo optical imaging," Proceedings of the National Academy of Sciences of the United States of America, vol. 102, no. 8, pp. 29222927, 2005.

[25] S. M. Evans, W. T. Jenkins, B. Joiner, E. M. Lord, and C. J. Koch, "2-Nitroimidazole (EF5) binding predicts radiation resistance in individual 9L s.c. tumors," Cancer Research, vol. 56, no. 2, pp. 405-411, 1996.

[26] M. E. Yildiz, R. K. Prud'homme, I. Robb, and D. H. Adamson, "Formation and characterization of polymersomes made by a solvent injection method," Polymers for Advanced Technologies, vol. 18, no. 6, pp. 427-432, 2007.

[27] F. Ahmed, R. I. Pakunlu, G. Srinivas et al., "Shrinkage of a rapidly growing tumor by drug-loaded polymersomes: $\mathrm{pH}$ triggered release through copolymer degradation," Molecular Pharmaceutics, vol. 3, no. 3, pp. 340-350, 2006.

[28] A. C. R. Grayson, M. J. Cima, and R. Langer, "Size and temperature effects on poly(lactic-co-glycolic acid) degradation and microreservoir device performance," Biomaterials, vol. 26, no. 14, pp. 2137-2145, 2005.

[29] T. Ivanova, I. Panaiotov, F. Boury, J. E. Proust, J. P. Benoit, and R. Verger, "Hydrolysis kinetics of poly(D,L-lactide) monolayers spread on basic or acidic aqueous subphases," Colloids and Surfaces B, vol. 8, no. 4-5, pp. 217-225, 1997.

[30] L. E. Gerweck and K. Seetharaman, "Cellular $\mathrm{pH}$ gradient in tumor versus normal tissue: potential exploitation for the treatment of cancer," Cancer Research, vol. 56, no. 6, pp. 1194$1198,1996$.

[31] R. J. Gillies, N. Raghunand, G. S. Karczmar, and Z. M. Bhujwalla, "MRI of the tumor microenvironment," Journal of Magnetic Resonance Imaging, vol. 16, no. 4, pp. 430-50, 2002, Erratum in: Journal of Magnetic Resonance Imaging, vol. 16, no. 6, pp.751, 2002.

[32] P. Swietach, R. D. Vaughan-Jones, and A. L. Harris, "Regulation of tumor $\mathrm{pH}$ and the role of carbonic anhydrase 9," Cancer and Metastasis Reviews, vol. 26, no. 2, pp. 299-310, 2007.

[33] N. A. Christian, M. C. Milone, S. S. Ranka et al., "Tatfunctionalized near-infrared emissive polymersomes for dendritic cell labeling," Bioconjugate Chemistry, vol. 18, no. 1, pp. 31-40, 2007. 
[34] N. A. Christian, F. Benencia, M. C. Milone et al., "in vivo dendritic cell tracking using fluorescence lifetime imaging and near-infrared-emissive polymersomes," Molecular Imaging and Biology, vol. 11, no. 3, pp. 167-177, 2009.

[35] L. H. Reddy, J. M. Renoir, V. Marsaud, S. Lepetre-Mouelhi, D. Desmaële, and P. Couvreur, "Anticancer efficacy of squalenoyl gemcitabine nanomedicine on 60 human tumor cell panel and on experimental tumor," Molecular Pharmaceutics, vol. 6, no. 5, pp. 1526-1535, 2009.

[36] L. W. Hertel et al., "Synthesis and biological activity of $2^{\prime}, 2^{\prime}$ difluorodeoxycytidine (gemcitabine)," in Biomedical Frontiers of Fluorine Chemistry, I. Ojima, J. R. McCarthy, and J. T. Welch, Eds., pp. 265-278, American Chemical Society, Washington, DC, USA, 1996. 

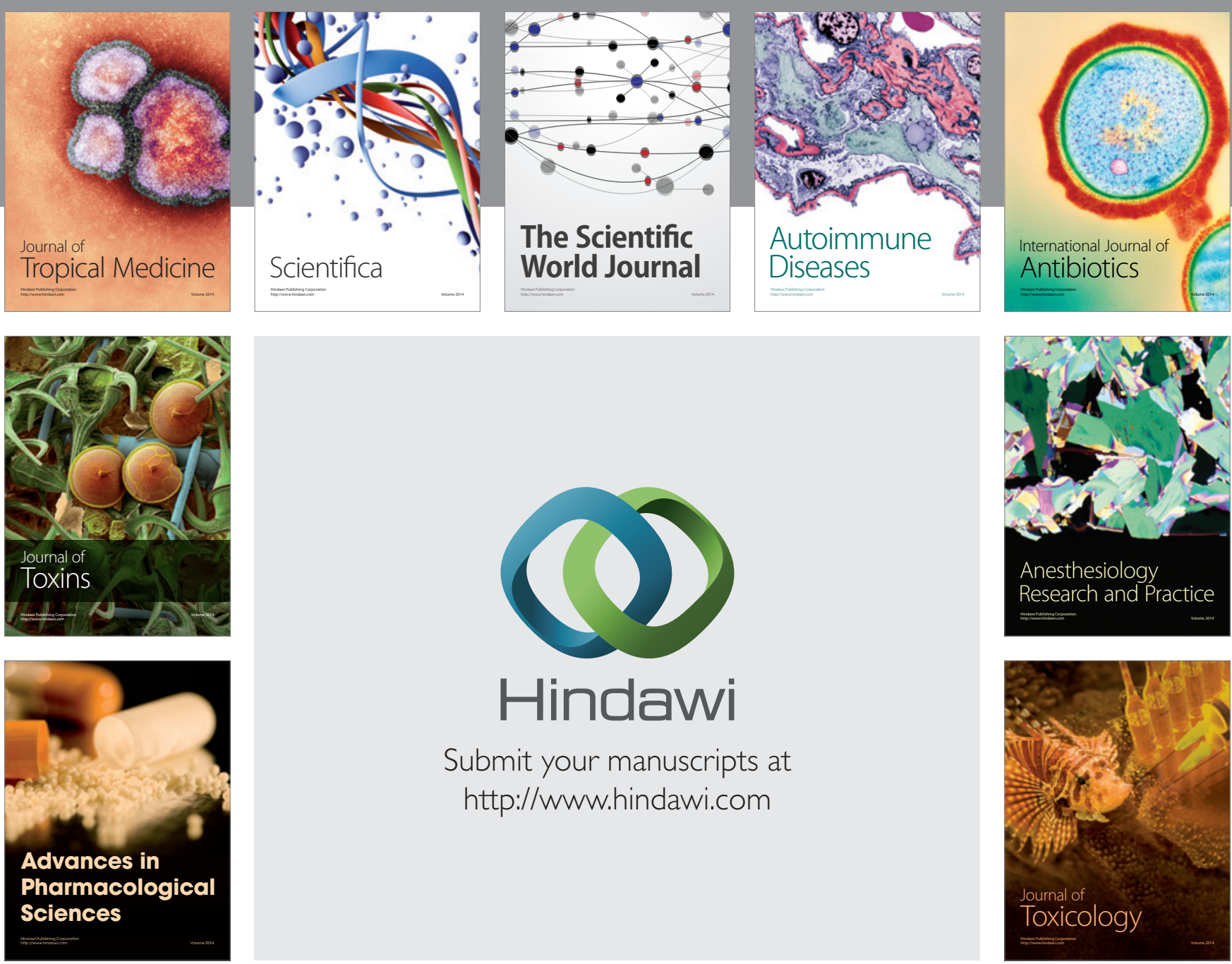

\section{Hindawi}

Submit your manuscripts at

http://www.hindawi.com
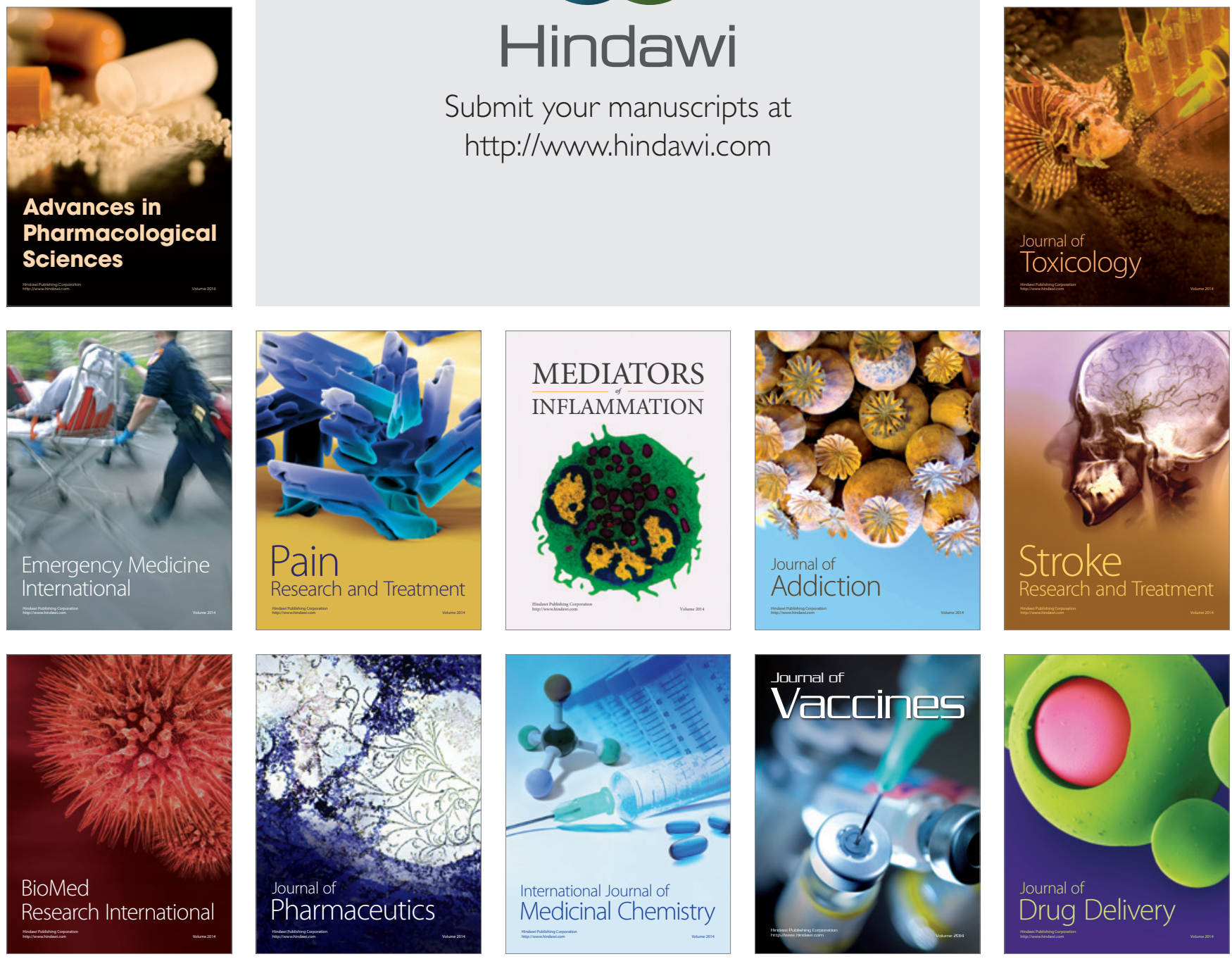